\title{
A Comparison of MAC Protocols for Ultrasonic Intra-body Sensor Networks
}

\author{
G. Enrico Santagati \\ Northeastern University, Boston, MA, U.S.A. \\ santagati.g@husky.neu.edu \\ Francesca Cuomo \\ "Sapienza" University of Rome, Italy \\ francesca.cuomo@uniroma1.it
}

\author{
Mirko Gradillo \\ "Sapienza" University of Rome, Italy \\ mirko.g@uni.net \\ Tommaso Melodia \\ Northeastern University, Boston, MA, U.S.A. \\ melodia@ece.neu.edu
}

\begin{abstract}
Wireless intra-body networks of implantable biomedical devices have the potential to enable revolutionary healthcare and clinical applications. In our previous work we investigated the use of ultrasonic waves as an alternative to radio frequency $(\mathrm{RF})$ waves as physical carrier of information, and proposed Ultrasonic WideBand (UsWB), the first ultrasonic integrated physical and medium access control (MAC) layer protocol.

In this paper, we compare the performance of the UsWB MAC protocol with two existing MAC protocols originally designed for wireless RF-based networks, ALOHA and Carrier Sense Multiple Access (CSMA). In particular, we discuss the protocol performance in terms of (i) average network throughput and packet drop rate, (ii) average short-term fairness, (iii) average packet delay and packet delay variation, and (iv) energy consumption per bit. We show that UsWB outperforms ALOHA in terms of throughput, while CSMA can achieve comparable performance under specific setups. However, both ALOHA and CSMA have very high packet drop rates as compared to UsWB. The latter is capable of keeping the packet drop rate under a pre-defined threshold. Moreover, UsWB significantly outperforms both ALOHA and CSMA in terms of short-term fairness, average packet delays and delay variation. Finally, CSMA has the highest energy consumption per bit, because of long idle sensing times, whereas UsWB has the lowest, and it can be further reduced by trading throughput for energy consumption through energy-minimizing rate adaptation.
\end{abstract}

\section{INTRODUCTION}

Wireless Intra-body Networks of implantable biomedical devices have the potential to enable revolutionary healthcare and clinical applications. Distributed remote cardiac

\footnotetext{
This material is based upon work supported by the National Science
} Foundation under grant CAREER CNS-1253309. rhythm monitors and wireless pacemakers could detect cardiovascular malfunctions and trigger preemptive measures to mitigate their effects. Continuous glucose monitoring in diabetic patients could enable reactive administration of insulin through under-skin miniaturized drug deliverers; while pill-sized ingestible cameras could provide an alternative to monitoring of the digestive tract based on intrusive examination techniques such as gastroscopy. Large amounts of physiological data captured by implantable sensors could also be forwarded to an out-of-body gateway such as a smartphone or a smartwatch, and be made available to physicians and patients through the Internet. Other potential applications enabled by networked implantable devices are countless and span in-vivo monitoring, reactive sensing and actuation, robotic microsurgery, and nano medicine.

Currently, existing wireless medical implants are connected through radio frequency ( $\mathrm{RF}$ ) electromagnetic waves. RFbased solutions tend to almost-blindly scale down traditional wireless technologies (e.g., Wi-Fi, Bluetooth, Zigbee) to the intra-body environment, with little or no attention to the peculiar characteristics and safety requirements of the human body. We contend that this is not the right approach. The human body is in fact composed of up to $65 \%$ water, a medium through which RF waves do not propagate well. In addition, the medical community is still divided on the risks caused by exposure of human tissues to RF radiation - the World Health Organization classifies RF waves as "possibly carcinogenic to humans". Moreover, RF-based technologies raise serious concerns with potential conflicts with existing RF communication systems that can unintentionally undermine the reliability and security of the intra-body network, and therefore the safety of the patient.

Given the limitations of RF-based solutions, in [1] we proposed and investigated the use of ultrasonic waves as an alternative carrier of information in human tissues. In $[2,3]$, we proposed Ultrasonic WideBand (UsWB), a new ultrasonic multipath-resilient physical and medium access control (MAC) layer integrated protocol. UsWB is based on the idea of transmitting short carrierless ultrasonic pulses following a pseudo-random adaptive time-hopping pattern, with a superimposed adaptive spreading code. We showed that the UsWB physical and medium access control protocols enable nodes to flexibly trade data rate performance for power consumption, and allow multiple concurrent users to coexist by dynamically adapting their transmission rate to channel and interference conditions.

As of today, and to the best of our knowledge, UsWB 
is the only MAC protocol specifically designed for ultrasonic intra-body sensor networks, while countless MAC protocols originally designed for traditional RF-based wireless networks are available. In this paper, we compare the performance of the UsWB MAC protocol with two existing random access MAC protocols originally designed for wireless RF-based networks, ALOHA and CSMA. In particular, we discuss the protocol performance in terms of (i) average network throughput and packet drop rate, (ii) average shortterm fairness, (iii) average packet delay and packet delay variation, and (iv) energy consumption per bit.

The remainder of the paper is organized as follows. In Section 2 we briefly discuss the fundamentals of ultrasonic communication in the human body. In Section 3 we introduce UsWB physical layer transmission scheme. In Section 4 we present the basic functionalities of the three MAC protocols under examination, and in Section 5 we thoroughly discuss their performance. Finally, in Section 6 we conclude the paper.

\section{ULTRASONIC INTRA-BODY COMMU- NICATIONS}

Ultrasounds are mechanical waves that propagate in an elastic medium at frequencies above the upper limit for human hearing, i.e., $20 \mathrm{kHz}$.

Attenuation. Two main mechanisms contribute to ultrasound attenuation in tissues, i.e., absorption and scattering. An initial pressure $P_{0}$ decays at a distance $d$ according to [4]

$$
P(d)=P_{0} e^{-\alpha d},
$$

where $\alpha$ (in $\left.\left[\mathrm{Np} \cdot \mathrm{cm}^{-1}\right]\right)$ is an amplitude attenuation coefficient that captures all the effects that cause dissipation of energy from the ultrasound wave. Parameter $\alpha$ depends on the carrier frequency through $\alpha=a f^{b}$, where $f$ represents the carrier frequency (in $\mathrm{MHz}$ ) and $a$ (in $\left[\mathrm{Np} \mathrm{m}^{-1} \mathrm{MHz}^{-\mathrm{b}}\right]$ ) and $b$ are attenuation parameters characterizing the tissue [1].

Propagation Speed. Ultrasonic wave propagation is affected by propagation delays that are orders of magnitude higher than RF. The propagation speed of acoustic waves in biological tissues is approximately $1500 \mathrm{~m} / \mathrm{s}$, as compared to $2 \times 10^{8} \mathrm{~m} / \mathrm{s}$ [5] for RF waves.

Reflections and Scattering. The human body is composed of different organs and tissues with different sizes, densities and sound speeds. Therefore, it can be modeled as an environment with pervasive presence of reflectors and scatterers. The direction and magnitude of the reflected wave depend on the orientation of the boundary surface and on the acoustic impedance of the tissues [1], while scattered reflections occur when an acoustic wave encounters an object that is relatively small with respect to its wavelength or a tissue with an irregular surface. Consequently, the received signal is obtained as the sum of numerous attenuated, perhaps distorted, and delayed versions of the transmitted signal.

Channel Modeling. In [2] we derive a deterministic channel model based on acoustic wave propagation theory. Propagation of acoustic waves through biological tissues is governed by three coupled first-order equations, i.e., the continuity equation, the force equation and the equation of state [6], which represent relationships among acoustic pressure $P$, acoustic particle velocity $u$, and medium density

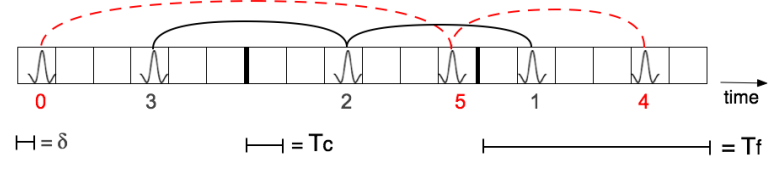

Figure 1: Two concurrent transmissions with $N_{h}=6$, $N_{s}=3$, time-hopping sequences $\{3,2,1\}$ and $\{0,5,4\}$ and spreading codes $\{1,1,-1\}$ and $\{1,-1,-1\}$.

$\rho$. A realistic model of ultrasonic propagation in human tissues that incorporates attenuation, scattering and multipath effect needs to simultaneously satisfy the three equations above. We solve these equation using a computationally efficient numerical approach based on the pseudo-spectral and k-space methods [7] to derive an accurate characterization of the channel impulse response, which can be used in PHY and MAC layer simulation studies. Further details are given in Section 5.1.

\section{UsWB PHYSICAL LAYER}

UsWB physical layer transmission scheme is based on the idea of transmitting short information-bearing carrierless ultrasonic pulses, following a pseudo-random adaptive time-hopping pattern with a superimposed spreading code of adaptive length. Impulsive transmission and spread-spectrum encoding combat the effects of multipath and scattering and introduce waveform diversity among interfering transmissions.

Consider, as in Fig. 1, a slotted timeline divided into slots of duration $T_{c}$, with slots organized in frames of duration $T_{f}=N_{h} T_{c}$, where $N_{h}$ is the number of slots per frame. Each user transmits one pulse per frame in a slot determined by a pseudo-random time-hopping sequence. Information is carried through pulse position modulation (PPM), i.e., a ' 1 ' symbol is carried by a pulse delayed by a time $\delta$ with respect to the beginning of the slot, while a '-1' symbol begins with the slot. Since a single pulse may collide with pulses transmitted by other users with a probability that depends on the frame size $N_{h}$, we represent each information bit with pseudo-orthogonal spreading codes of variable length, $N_{s}$ because of (i) their excellent and well-understood multiple access performance, (ii) limited computational complexity, and (iii) inherent resilience to multipath. The resulting transmitted signal for a symbol $d$ can be modeled as

$$
s(t)=\sum_{j=0}^{N_{s}-1} p\left(t-c_{j} T_{c}-j T_{f}-\frac{a_{j} d+1}{2} \delta\right)
$$

where $p(t)$ is the pulse shape, $\left\{c_{j}\right\}$ is the time-hopping sequence with $0 \leq c_{j} \leq N_{h}-1,\left\{a_{j}\right\}$ is the pseudo-orthogonal spreading code of $N_{s}$ chips with $a_{j} \in\{-1,1\}$, and $\delta$ is the PPM shift of a pulse representing a ' 1 ' chip.

\section{MAC PROTOCOLS}

UsWB Rate Adaptation . The low-duty-cycle impulsebased transmission scheme with a superimposed spreading code allows multiple transmitters to coexist on the same channel. In UsWB, by dynamically and distributively adapting their time-hopping frame length and spreading code length, multiple users coexist without the need for mutual temporal exclusion among different transmissions. By adapting frame and code length, users control the tradeoffs among (i) resilience to multi-user interference and ultrasonic channel 
errors, (ii) achievable information rate, and (iii) energy efficiency. As discussed in detail in [2], by controlling the timehopping frame length $N_{h}$, i.e., the average inter-pulse time, a user can adapt the transmission rate (which decreases with larger time-hopping frame), and as a consequence modify the average radiated power and therefore the level of interference generated to other ongoing communications. By controlling $N_{s}$, i.e., the number of pulses per information bit, a user can control the tradeoff between robustness to multi-user interference and noise (which increases with longer spreading codes), energy consumption per bit (which increases linearly with increasing $N_{s}$ ) and information rate (decreasing with increasing $N_{s}$ ). UsWB optimally, distributively, and asynchronously regulates these tradeoffs to (i) maximize the communication rate, or (ii) minimize the energy consumption.

In this work, we consider the rate-maximizing adaptation in [2], where each user distributively maximizes its transmission rate by selecting an optimal pair of code and frame lengths based on the current level of interference and channel quality for a given maximum tolerable BER. Rate adaptation is achieved through an ad-hoc designed protocol. A twoway handshake opens the connection between two nodes, $T_{x}$ and $R_{x}$. Once the connection has been established, the receiver $R_{x}$ estimates the interference and calculates the frame and spreading code lengths that maximize the communication throughput, as discussed in detail in [2]. This information is piggybacked into ACK or NACK packets.

ALOHA. ALOHA is a random access protocol where nodes do not check whether the channel is busy or idle before transmitting [8]. Nodes that want to transmit data simply access the channel and transmit the data. If the packet is successfully received, the receiver sends an ACK packet to the transmitter after waiting for a small time interval, i.e., a Short Inter-Frame Space (SIFS). If no acknowledgment is received, i.e., a collision or a transmission error has occurred, the transmitter attempts a retransmission after a random time interval. This randomness prevents collisions from recurring indefinitely. The backoff time is selected randomly in the range between zero and a maximum backoff time. Selecting the maximum backoff is not trivial. In fact, low values of maximum backoff may still lead to high probability of collision, thus lower throughput. Conversely, large maximum backoff times may introduce unnecessary idle times on the channel, therefore increasing the average packet delay and reducing the system fairness.

We consider a maximum backoff given as $B_{\max } \cdot T_{s}$, where $T_{s}$ is the time for a node to receive an ACK packet, i.e., transmission time and maximum propagation delay in the considered topology, and $B_{\max }$ is an integer maximum backoff variable that is varied in a given range.

Carrier Sense Multiple Access. We consider a 1persistent CSMA with Collision Avoidance (CA) protocol with physical carrier sensing [9]. CSMA/CA is a multiple access technique based on carrier detection, which allows multiple nodes to share the channel by avoiding simultaneous transmissions, therefore avoiding collisions among transmitted packets. When a node wants to transmit a data packet, it first listens to the channel. If the channel is sensed as idle during a fixed time interval, i.e., Distributed Inter-Frame Space (DIFS), the node transmits. If the transmission is successful, the receiver sends an ACK packet after a SIFS. If no acknowledgment is received the transmitter schedules a

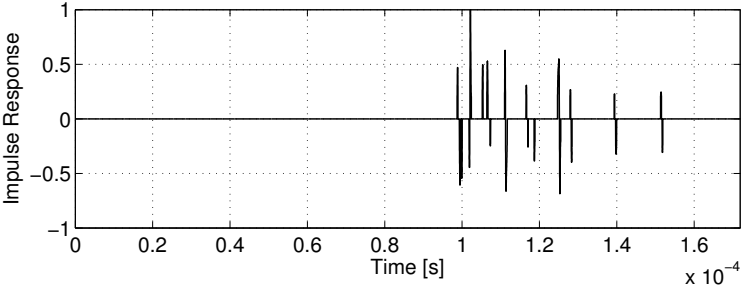

Figure 2: Ultrasonic normalized channel impulse response in the modeled 2-D human arm.

retransmission after a backoff time. During this process, the other nodes sense the channel as busy, and do not initiate new transmissions.

When a transmitting node senses the channel as busy, it waits for a backoff time during which it keeps checking the status of the channel. The backoff time is drawn from a uniform distribution in $[0, C W-1]$, where $C W$ denotes the contention window. The backoff timer is decremented every single period of channel inactivity, i.e., every time the channel is sensed as idle. When the backoff timer expires, the station attempts a new transmission with probability 1 , i.e., 1-persistent mode. CSMA is based on a binary exponential backoff mechanism. The $C W$ value is initially set to a minimum value $C W_{\min }$ and it is doubled after each collision, up to a maximum value $C W_{\max }$. $C W$ value is halved after each successful transmission until it reaches $C W_{\text {min }}$.

Finally, because of the carrierless nature of the physicallayer transmission scheme, CSMA does not use a traditional clear channel assessment (CCA) based on carrier sensing. Instead, we assume a pulse sensing CCA that uses a simple threshold-based energy detector to detect whether the channel is idle or not [10].

\section{PERFORMANCE EVALUATION}

\subsection{Multi-scale Simulator}

In this section, we evaluate the performance of the three MAC protocols discussed in Section 4 through a customdesigned multi-scale simulator [2] that models the ultrasonic communication performance at three different levels: (i) at the acoustic wave level by capturing ultrasonic propagation in tissues through reflectors and scatterers, (ii) at the bit level by simulating in detail the physical layer transmission scheme, (iii) at the packet level by simulating networked operations and distributed medium access control.

Acoustic wave level. The acoustic wave level simulations are based on a Matlab toolbox, i.e., k-Wave [11], which implements the pseudo-spectral and k-space method, discussed in Section 2, to solve acoustic wave propagation equations. The simulation consists of a 2-dimensional section of the human arm, including bones, muscles, fat and skin, as in [2]. We recorded a channel impulse response by simulating the propagation of an omnidirectional ideal Dirac pulse in the medium. In Fig. 2 we show the resulting channel impulse response. We observe that multipath and scattering effects introduce attenuated signal replicas spaced in time. Because of the very short duration of the transmitted pulses, replicas do not interact destructively. The simulated channel impulse response is used as channel model in the upper layer simulator.

Bit level. Transmission at the bit level is modeled through a custom physical layer simulator, which produces as out- 

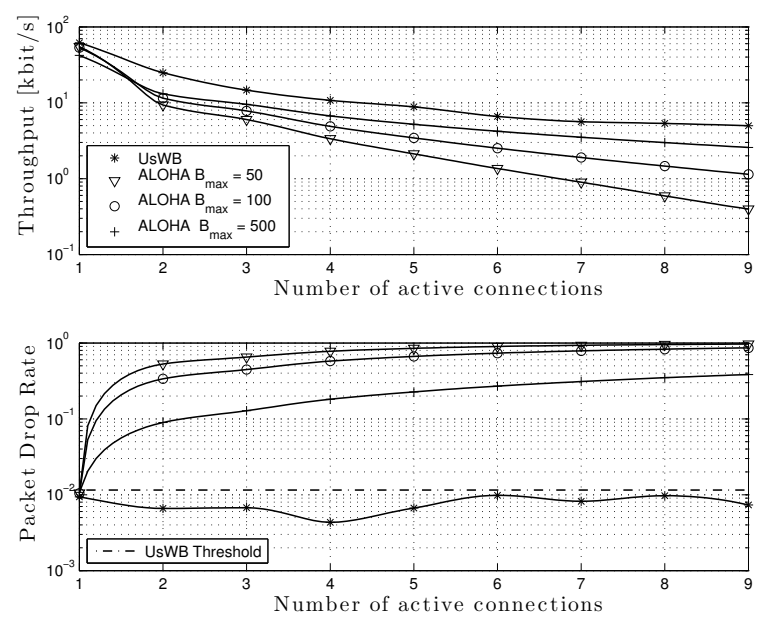

Figure 3: UsWB vs ALOHA: Average network throughput (top) and Packet Drop Rate (bottom) as a function of the number of active connections.

put an empirical model of the BER against different values of the time-hopping frame length and spreading code length, for different levels of interference. The physical layer simulation models a transmitter and a receiver communicating over an ultrasonic channel with the UsWB transmission scheme discussed in Section 3. Simulations are performed to obtain an estimate of the achievable BER upon varying the frame length and the spreading code length with a different number of interferers transmitting on the same channel. In the simulations we neglect both frame and time hopping synchronization errors so that the BER only considers interference effects. We enhanced the bit-level simulator in [2] with a more accurate model of the inter-symbolic interference (ISI). An extensive simulation campaign was conducted and BER values were obtained as a function of the spreading code and frame lengths for different number of simultaneously active connections.

Network level. The empirical model of the BER as a function of frame length and spreading code length for a given level of interference is then imported in a Javabased event-driven packet-level simulator, which models the UsWB, ALOHA and CSMA MAC protocol functionalities. We consider a 2-D topology with 18 static nodes randomly located inside a square of side length $40 \mathrm{~cm}$. Each transmitter node communicates with a randomly-selected receiver node, in a point-to-point and single-hop fashion, resulting in a maximum of nine simultaneously communicating pairs. We also assume that the transmission range is greater than the maximum distance between the nodes. Thus, all nodes are affected by the same number of interferers.

The simulation time is set to $100 \mathrm{~s}$. We consider an infinite arrival rate at each transmitter, i.e., transmitters are always backlogged. The maximum allowed frame and spreading code length is set to 15 slots and 20 chips, respectively. The maximum supported rate, achieved when frame and spreading code length are both set to one, is equal to $2 \mathrm{Mbit} / \mathrm{s}$, i.e., the slot duration $T_{c}$ is fixed to $5 \mu \mathrm{s}$. The data packet size is fixed to 145 byte, with 20 byte being the packet header. The ACK and NACK packets consist only of the 20 byte header. We set the maximum tolerable BER to $10^{-5}$, which results in approximately $10^{-2}$ maximum tolerable packet drop rate for data packets.
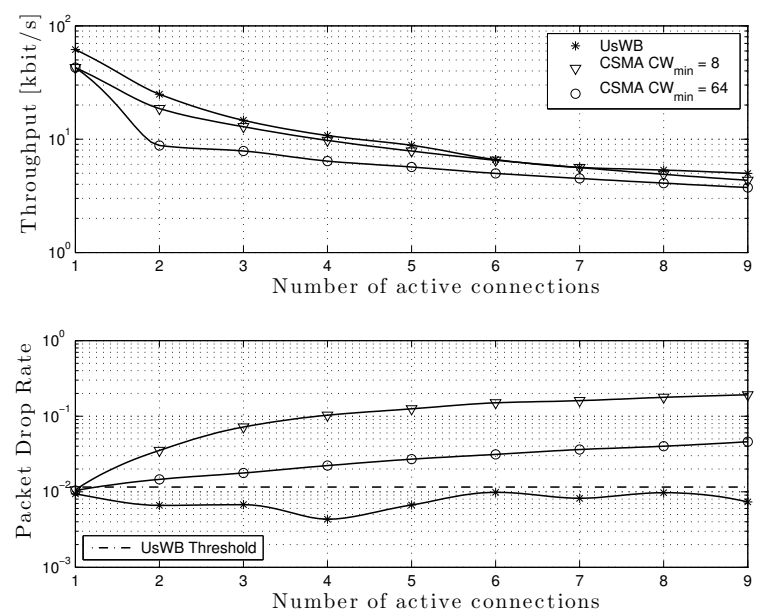

Figure 4: UsWB vs CSMA: Average network throughput (top) and Packet Drop Rate (bottom) as a function of the number of active connections.

For the UsWB simulations, we consider the rate-maximizing solution of the implicitly cooperative problem [2]. Accordingly, during the simulations, frame length and spreading code length are adapted to the interference level measured at the receiver to maximize the node data rate while satisfying the maximum packet drop rate requirement. For CSMA and ALOHA, frame length and spreading code length are fixed to satisfy the BER constraint of $10^{-5}$ against channel condition and ISI only, i.e., with no interference. The interference effect is mitigated by random backoff and CCA techniques, in ALOHA and in CSMA, respectively. Moreover, we consider the random backoff expressed in integer values as number of time periods. The time period is defined as the time for a node to receive an ACK packet, which includes both the transmission delay and the propagation delay, which is not negligible because of the low speed of sound in biological tissues. Based on this, in ALOHA we vary $B_{\max }$ between 50 and 500, while in CSMA we fix $C W_{\max }=256$ and we vary $C W_{\min }$ between 8 and 64 .

In the following, we discuss and compare the performance of three MAC protocols in terms of (i) average network throughput and packet drop rate, (ii) average short-term fairness, (iii) average packet delay and packet delay variation, and (iv) energy consumption per bit.

\subsection{Simulation Results}

Throughput and Packet Drop Rate. We define the throughput as the average rate of information correctly received during the simulation time per active connection. The packet drop rate is defined as the ratio between the number of packets dropped and the number of packets that reach the top of the transmitter queue, averaged over all the active connections. Both performance metrics are evaluated as a function of the number of active connections in the channel.

In Figs. 3 and 4, we compare UsWB network throughput (top) and packet drop rate (bottom) with ALOHA and CSMA protocols, respectively, for different values of $B_{\max }$ and $C W_{\min }$. We observe that by increasing the maximum backoff in ALOHA the throughput increases, while the packet drop rate decreases. In fact, a larger backoff time allows nodes to find larger time intervals with no interference, in which they can capture the channel and transmit bursts of 

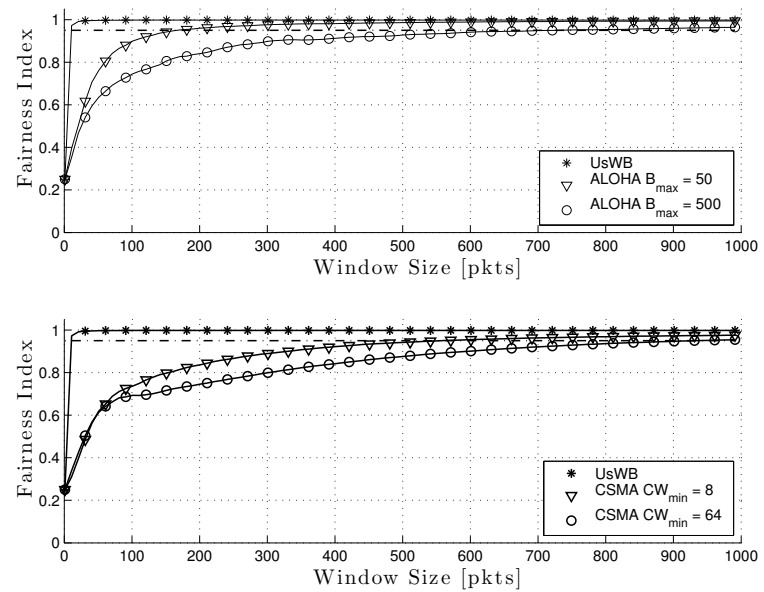

Figure 5: UsWB vs ALOHA (top) and UsWB Vs CSMA (bottom): Average Jain fairness index for 4 simultaneously communicating pairs as a function of the window size.

packets with no collisions. However, for a $B_{\max }$ up to 500, UsWB still guarantees higher throughput. Note that, when only one pair is communicating, a longer backoff time in ALOHA protocol does not positively contribute. Instead, this increases the idle times and therefore reduces the connection throughput. Differently, by increasing $C W_{m i n}$, CSMA protocol has a lower throughput, and the packet drop rate also decreases. In fact, a larger minimum contention window leads nodes to select higher backoff intervals as soon as a collision is detected. Although this still leads to the channel capture phenomenon discussed above that reduces the probability of collision, it inevitably introduces longer idle times that lead to channel underutilization. Finally, by comparing the protocols in terms of packet drop rates, we can show that the UsWB protocol always satisfies the maximum packet drop rate threshold of approximately $10^{-2}$. Since the UsWB optimization variables, i.e., code and frame length, assume integer values only, the packet drop rate in UsWB presents a fluctuating behavior. In fact, although the BER experienced at the receiver is always lower than the maximum tolerable BER constraint, i.e., $10^{-5}$, the exact value of $\mathrm{BER}$, hence the packet drop rate, given by the optimal pairs for different numbers of active connections is not necessarily constant. These BER variations for different numbers of active connections will also affect the delay, the delay variation and the bit-cost of the UsWB protocol.

Short-Term Fairness. Fairness measures how fair the distribution of throughput among nodes is. We evaluate the three protocols' fairness using the Jain fairness index. The Jain fairness index is defined as

$$
F I=\frac{\left(\sum_{i=1}^{|\mathcal{N}|} x_{i}\right)^{2}}{|\mathcal{N}| \sum_{i=1}^{|\mathcal{N}|} x_{i}^{2}},
$$

where $|\mathcal{N}|$ is the number of active connections and $x_{i}$ is the throughput of the $i^{\text {th }}$ connection.

We analyze the fairness protocol performance studying the distribution of network resources over short time scales, i.e., short-term fairness. Short-term unfairness can produce significant performance degradation for applications and upper layer protocols [12]. We use a sliding window method to measure the short-term fairness of the three protocols

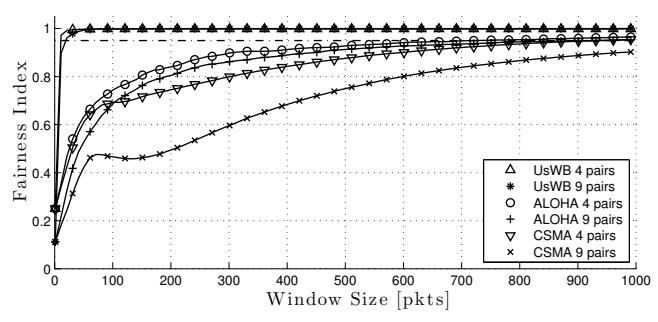

Figure 6: UsWB vs ALOHA vs CSMA: Average Jain fairness index for 4 and 9 simultaneously communicating pairs as a function of the window size.

that consists in calculating the Jain fairness index over a progressively increasing time scale [12].

In Fig. 5, we compare UsWB with ALOHA (top) and CSMA (bottom) in terms of short-term fairness when 4 pairs of nodes are simultaneously communicating. To this purpose, we plot the Jain fairness index as a function of the sliding window size. We consider as window size the overall number of packets correctly received in the network. In general, we can say that for a fixed number of communicating pairs UsWB protocol is fairer than the ALOHA protocol, which, in turn, performs better than CSMA protocol. In particular, we observe that lower $B_{\max }$ and $C W_{\min }$ values result in higher fairness, since they reduce the capture effect discussed before. In fact, when lower backoff times are selected, all the nodes can attempt to access the channel more often, thus increasing the probability of collision, but also increasing the overall system fairness. For example, by setting a threshold to 0.95 , i.e., a value over which we considered the system to be fair, we observe that UsWB reaches this target almost immediately, i.e., a window size smaller than 10 packets. In ALOHA with $B_{\max }=50$ and CSMA with $C W_{\min }=8$, fairness is achieved for window sizes larger than approximately 150 and 500 packets, respectively.

Figure 6 compares the short-term fairness of the three protocols for 4 and 9 communicating pairs, fixing $B_{\max }=500$ and $C W_{\min }=8$. In ALOHA and CSMA, we observe that by increasing the number of active connections the fairness decreases. By increasing the number of connections the probability of collision also increases, and this leads nodes with collisions to select a larger $\mathrm{CW}$ than the nodes that have successfully transmitted. As a consequence, the nodes in the latter group will be favored in future transmissions resulting in lower system fairness.

Packet Delay. We define as average packet delay the time between the moment when a packet reaches the head of the transmitter's queue to the moment in which it is successfully decoded.

In Fig. 7, we compare UsWB with ALOHA (top) and CSMA (bottom) in terms of average packet delay as a function of the number of active connections. We observe that UsWB packet delay is lower than packet delay in CSMA and ALOHA protocols. Moreover, increasing $C W_{\min }$ and $B_{\max }$ leads to higher packet delay, because the probability of selecting a larger backoff increases. Figure 7 also shows how the transmission delay component contributes to the total packet delay. We observe that UsWB transmission delay almost coincides with the total packet delay, while for ALOHA and CSMA the transmission delay is just a small fraction of the total packet delay. This behavior is caused by the idle times and frequent retransmissions that occur in 

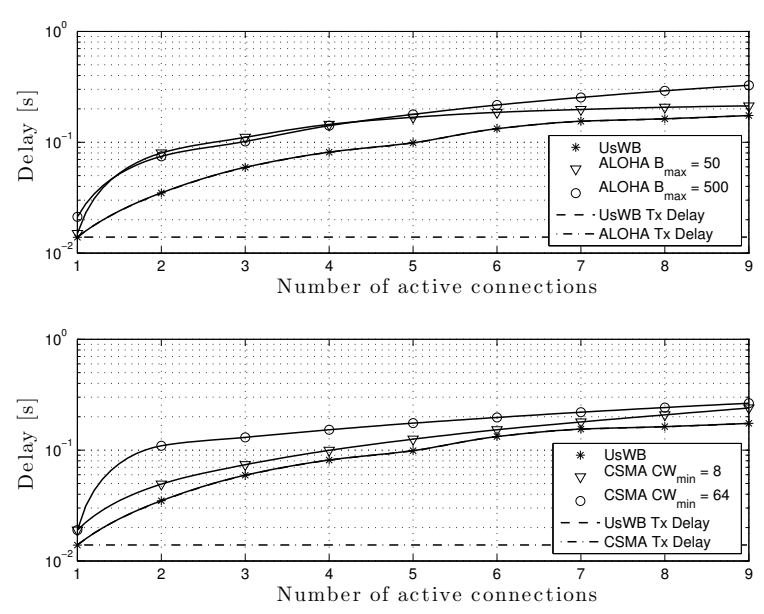

Figure 7: UsWB vs ALOHA (top) and UsWB vs CSMA (bottom): Average packet delay as a function of the number of active connections.

ALOHA and CSMA, especially for a higher number of active connections. The UsWB protocol, instead, by adapting time-hopping frame and code length, allows nodes to communicate simultaneously with low packet drop rate, i.e., few retransmissions, and enables MAC functionalities without mutual temporal exclusion, i.e., very low idle times.

Energy per Bit. We define the bit-cost $B_{c}$ as the average amount of energy that a node spends to successfully transmit one information bit. This can be obtained as the ratio between the total average energy spent by a node $E_{t o t}$ and the number of information bits successfully transmitted $B_{s}$. By decomposing the average total energy, the bit-cost can be expressed as

$$
B_{c}=\frac{P_{t x} T_{t x}+P_{r x} T_{r x}+P_{l i s t} T_{l i s t}}{B_{s}},
$$

where $P_{t x}, T_{t x}, P_{r x}, T_{r x}, P_{l i s t}$ and $T_{\text {list }}$ represent the average powers and times spent by the node to transmit, receive, and idle listening the channel, respectively.

The transmission component of the total energy is the energy consumption caused by the transmission of all bits, including retransmissions and header bits. The reception component represents the amount of energy consumed to receive the bits in the acknowledgement packets. Assuming that the transceiver can go into a low-power sleep-mode between consecutive pulse transmissions and receptions, $P_{t x}$ and $P_{r x}$ can be obtained as the energy required for transmitting and receiving one pulse times the number of pulse transmitted and received in one second

$$
P_{t x, r x}=\frac{E_{b, t x / r x}}{N_{h} T_{c}},
$$

where $N_{h}$ and $T_{c}$ are the time-hopping frame length and the chip duration, respectively, as in Section 3. $E_{b, t x}$ and $E_{b, r x}$ are the energy required to transmit and receive a pulse, respectively. Note that $N_{h}$ is fixed in ALOHA and CSMA, while it is adapted to the channel condition and interference level in UsWB. Finally, the idle listening energy consumption is the energy consumed by a node to sense the channel, either waiting for an acknowledgment packet, or checking whether the channel is idle or not. In this idle listening state, the node listens continuously to the channel, and the transceiver is never switched to the sleep mode. In UsWB
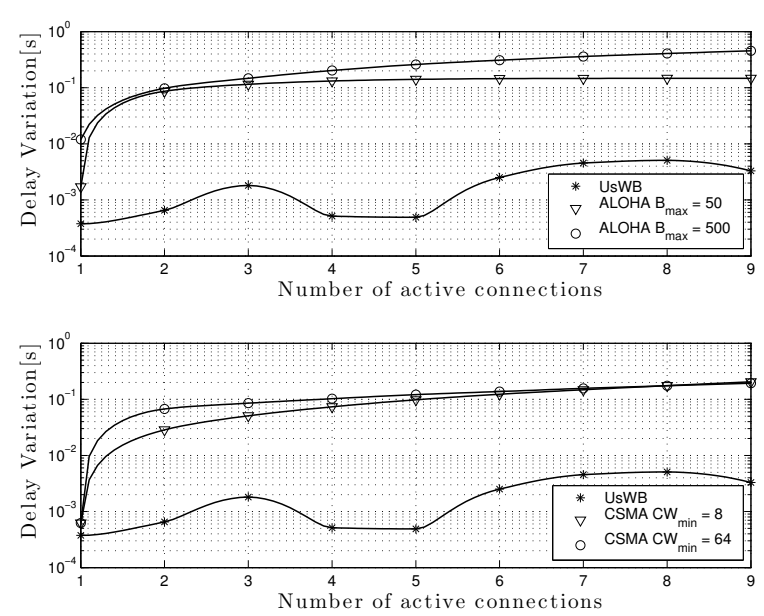

Figure 8: UsWB vs ALOHA (top) and UsWB vs CSMA (bottom): Average packet delay variation as a function of the number of active connections.
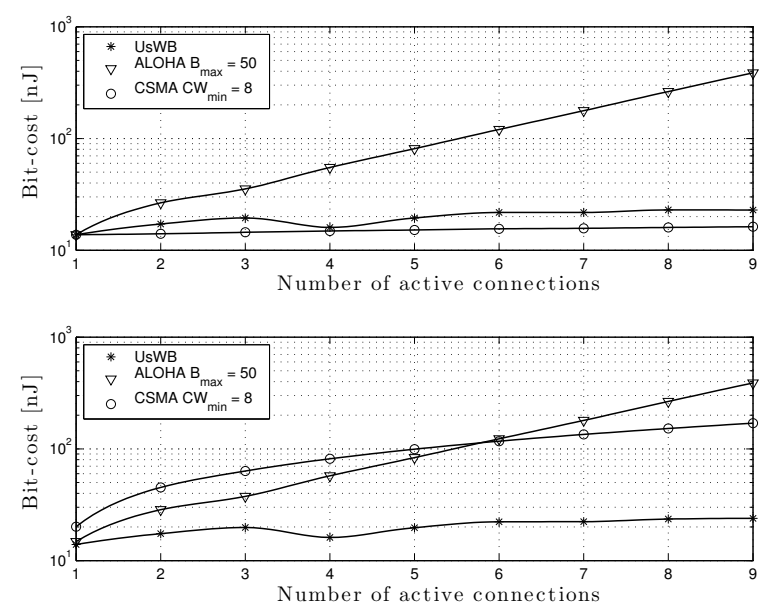

Figure 9: UsWB vs ALOHA vs CSMA: Bit-cost as a function of the number of active connections for $P_{\text {list }}=0$ (top) and $P_{\text {list }}=1 \mathrm{~mW}$ (bottom).

and ALOHA, the transmitting node listens to the channel only after a packet transmission, while waiting for an ACK packet to be received. However, In ALOHA, the listening time is slightly longer than in UsWB, because the receiver waits for a SIFS interval before transmitting the ACK packet. Also, by using NACK packets, UsWB can further reduce the listening time in case of transmission failures. In CSMA, nodes sense the channel before transmitting and while waiting for acknowledgments.

In Fig. 9 we compare the bit-cost for the three protocols as a function of the number of active connections, assuming $P_{\text {list }}=0$ (top), i.e., no idle listening energy consumption, and $P_{\text {list }}=1 \mathrm{~mW}$ (bottom), for $E_{b, t x}$ and $E_{b, r x}$ fixed to $1 \mathrm{~nJ}$. We observe that with $P_{\text {list }}=0$ the bit-cost is mainly due to the transmission energy consumption. Under this condition the CSMA protocol has lower bit-cost than ALOHA and UsWB. The high energy consumption of ALOHA is caused by the high packet drop rate and the resulting high number of retransmissions. The bit-cost of UsWB depends on the number of pulses per bit, i.e., the code length $N_{s}$, which increases with the number of active connections as a result of the rate-maximizing adaptation, 

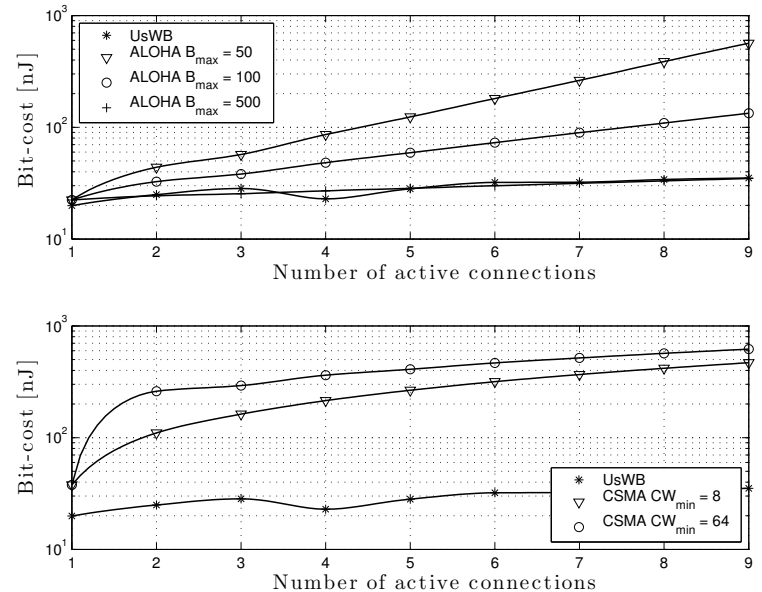

Figure 10: UsWB vs ALOHA (top) and UsWB vs CSMA (bottom): Bit-cost as a function of the number of active connections.

resulting in a higher transmission energy consumption. Note that the UsWB bit-cost can be lowered by changing the objective of the rate adaptation, i.e., by adapting the rate based on the energy-minimizing strategy discussed in detail in [2]. However, when energy consumption caused by idle listening is considered, i.e., $P_{\text {list }}=1 \mathrm{~mW}$, the bit-cost for CSMA increases significantly, while the bit-costs of ALOHA and UsWB are substantially the same. This is due to the fact that very little idle listening is performed in ALOHA and UsWB as compared to CSMA.

Figure 10 compares UsWB with ALOHA (top) and CSMA (bottom) in terms of bit-cost as a function of the number of active connections in the network, for different values of $B_{\max }$ and $C W_{\min }$. We consider as $E_{b, t x}, E_{b, r x}$ and $P_{\text {list }}$ the energy values of a transceiver designed for impulse-radio communications [13], i.e., $E_{b, t x}=1.44 \mathrm{~nJ}, E_{b, r x}=1.16 \mathrm{~nJ}$ and $P_{\text {list }}=2.9 \mathrm{~mW}$. We observe that, since the bit-cost of ALOHA proportionally depends on the packet drop rate, it can be reduced by assuming higher $B_{\max }$, hence by paying the price of higher delays and lower fairness. Finally, since the idle listening energy consumption is the larger component of the CSMA bit-cost, the bit-cost and average delay curves follow similar patterns, i.e., increasing with higher values of $C W_{\text {min }}$. In fact, the CSMA backoff times that lead to high delays also lead to long idle listening intervals, and therefore to high energy consumption.

\section{CONCLUSIONS}

We compared the performance of the UsWB MAC protocol with two existing MAC protocols originally designed for wireless RF-based networks, ALOHA and CSMA. In particular, we discussed the protocol performance in terms of (i) average network throughput and packet drop rate, (ii) average short-term fairness, (iii) average packet delay and packet delay variation, and (iv) energy consumption per bit.

We showed that UsWB outperforms ALOHA in terms of throughput, while CSMA can achieve comparable performance under specific setups. However, both ALOHA and CSMA have very high packet drop rates as compared to UsWB, which always keeps the packet drop rate under a given threshold. Moreover, UsWB clearly performs better than both ALOHA and CSMA in terms of short-term fairness, average packet delay and delay variation. Finally, CSMA has the highest energy consumption per bit because of the long idle listening times. UsWB bit-cost is the lowest, and can be further reduced by trading throughput for energy consumption through energy-minimizing rate adaptation.

\section{REFERENCES}

[1] L. Galluccio, T. Melodia, S. Palazzo, and G. E. Santagati. Challenges and Implications of Using Ultrasonic Communications in Intra-body Area Networks. In Proc. of IEEE Intl. Conf. on Wireless On-demand Networked Systems (WONS), Courmayeur, Italy, January 2012.

[2] G.E. Santagati, T. Melodia, L. Galluccio, and S. Palazzo. Medium access control and rate adaptation for ultrasonic intra-body sensor networks. IEEE/ACM Transactions on Networking, 2014.

[3] G. E. Santagati and T. Melodia. Sonar Inside Your Body: Prototyping Ultrasonic Intra-body Sensor Networks. In Proc. of IEEE Conf. on Computer Communications (INFOCOM), Toronto, Canada, April 2014.

[4] C. R. Hill. Ultrasonic attenuation and scattering by tissues. John Wiley \& Sons, Inc., 1978.

[5] Frank P. Bolin, Luther E. Preuss, Roy C. Taylor, and Robert J. Ference. Refractive index of some mammalian tissues using a fiber optic cladding method. Appl. Opt., 28(12):2297-2303, Jun. 1989.

[6] D. T. Blackstock. Fundamentals of Physical Acoustics. Wiley-Interscience, Feb. 2000.

[7] B. T. Cox, S. Kara, S. R. Arridge, and P. C. Beard. k-space propagation models for acoustically heterogeneous media: Application to biomedical photoacoustics. Acoustical Society of America, 121(6), Feb. 2007.

[8] Norman Abramson. The ALOHA system: Another alternative for computer communications. In Proc. of the 1970 Fall Joint Computer Conference, AFIPS, Houston, Texas, Nov. 1970.

[9] L. Kleinrock and F.A. Tobagi. Packet switching in radio channels: Part i-carrier sense multiple-access modes and their throughput-delay characteristics. IEEE Transactions on Communications, 23(12):1400-1416, Dec. 1975.

[10] N.J. August, Hyung-Jin Lee, and Dong-Sam Ha. Pulse sense: a method to detect a busy medium in pulse-based ultra wideband (UWB) networks. In Proc. 3th Ultra Wideband System and Technologies (UWBST), pages 366-370, May 2004.

[11] B. Treeby and B. Cox. k-wave. Technical report, http://www.k-wave.org/.

12] Can Emre Koksal, Hisham Kassab, and Hari Balakrishnan. An analysis of short-term fairness in wireless media access protocols (poster session). In Proc. of the Intl. Conf. on Measurement and Modeling of Computer Systems (SIGMETRICS), Santa Clara, California, USA, 2000.

[13] M. Crepaldi, Chen Li, J.R. Fernandes, and P.R. Kinget. An ultra-wideband impulse-radio transceiver chipset using synchronized-ook modulation. IEEE Journal of Solid-State Circuits, 46(10):2284-2299, Oct. 2011. 